\section{Osteopathie}

Offizielles Organ folgender Verbände

Verband der Osteopathen Deutschland e.V. (VOD)

Deutsche Akademie für Osteopathische Medizin e.V. (DAOM)

Dssociation Luxembourgeoise des Ostéopathes (A.L.D.O.)

ISSN $1610-5044$

2003; 1 (1)

Herausgeber :

Prof. Dr. rer. nat. med. habil. Rainer Breul D.O.h.c.

Marina Fuhrmann, D.O. M.R.O.

Prof. Dr. med. habil. Karl-Ludwig Resch

Dr. med. Roger Seider, D.O.

Verlag

Hippokrates Verlag in

MVS Medizinverlage Stuttgart GmbH \& Co.KG

Steiermärker Str. 3-5, 70469 Stuttgart, Fax: (07 11) 8931-706

Geschäftsführung:

Dr. med. Thomas Scherb, Dipl. Kaufm. Albrecht Hauff

Programmplanung:

Marina Horbatsch

Fon/Fax: (07 11) 89 31-715/-705

E-Mail: marina.horbatsch@medizinverlage.de und

redaktion.do@medizinverlage.de

Redaktion:

Christoph Newiger in team 4 U Medienbüro

Fon/Fax: (089) 209001-67/-68

E-Mail: redaktion.do@medizinverlage.de

Beratung:

Dorothee Seiz

in MVS Medizinverlage Stuttgart GmbH \& Co. KC

E-Mail: redaktion.do@medizinverlage.de

Marketing:

Marion Krubasik

Fon/Fax: (07 11) 89 31-735/-706

E-Mail: marion.krubasik@medizinverlage.de

Anzeigen:

Günter Fecke, MVS Media-Service

Fon/Fax: (07 11) 8931-714/-706

E Mail: guenter.fecke@medizinverlage.de

Abonnenten-Service

Fon/Fax: (07 11) 8931-321/-422

E Mail: kundenservice@thieme.de

Produktion

Satz und Gestaltung: Fotosatz Sauter, Donzdorf

Druck: Rondo Druck, Ebersbach

Urheberrecht:

Mit der Annahme eines Manuskriptes erwirbt der Verlag für die Dauer der gesetzlichen Schutzfrist (§64 UrhRG) die ausschließliche Befugnis zur Wahrnehmung der Verwertungsrechte im Sinne der $\S \S 15 \mathrm{ff}$. des Urheberrechtsgesetzes. Die Zeitschrift und alle in ihr enthaltenen einzelnen Beiträge und Abbildungen sind für die Dauer des Urheberrechts geschützt. Jede Verwertung ist ohne Zustimmung des Verlages außerhalb der engen Grenzen des UrhRG unzulässig und strafbar.

Dies gilt insbesondere für Vervielfältigungen, Übersetzungen, Microverfilmungen und die Einspeicherung und Verarbeitung in elektronischen Systemen. Für den persönlichen Gebrauch dürfen von Beiträgen oder Teilen von diesen einzelne Kopien hergestellt werden. Die Rechte an den Abbildungen liegen - wenn nicht anders gekennzeichnet - beim Verlag.

Erscheinungsweise:

Vierteljährlich

Bezugspreise

$\begin{array}{llll} & \begin{array}{l}\text { Abonnement- } \\ \text { preis }\end{array} & \begin{array}{l}\text { Versand- } \\ \text { kosten }\end{array} & \text { Gesamt } \\ \text { Inland } & € 64,90 & € 5,90 & € 70,80 \\ \text { Europa } & € 64,90 & € 10,40 & € 75,30 \\ \text { Restl. Welt } & € 64,90 & € 18,90 & € 83,80\end{array}$

Aus-, Fort- und Weiterbildungspreis/Vorzugspreis für Studenten und AIP:

$\begin{array}{llll}\text { Inland } & € 44,90 & € 5,90 & € 50,80 \\ \text { Europa } & € 44,90 & € 10,40 & € 55,30 \\ \text { Restl. Welt } & € 44,90 & € 18,90 & € 63,80\end{array}$

Einzelheft $€ 18,00$ zzgl. Versandkosten ab Verlagsort. Alle Preise sind unverbindlich empfohlene Preise. Alle Preise und Versandspesen enthalten 7\% MwSt.

\title{
Ist der Osteopath der bessere Orthopäde?
}

S chon aus den KG-/Physiotherapie-Praxen hörte man es häufig, und seit die Osteopathie immer weitere Verbreitung findet nimmt es zu: „Ärzte haben ja keine Ahnung!“ Das gilt besonders für die Spezialisten am Bewegungsapparat, die Orthopäden. Klar, man erzählt gerne, dass die Facharztausbildung fast ausschließlich operativ ausgerichtet ist, dass in den Praxen Patienten häufig im 2-Minuten-Takt durchgeschleust werden ohne adäquate Untersuchung. Funktionelle Sachverhalte sind ihnen weitgehend unbekannt. Dagegen kennen sich doch die Osteopathen wirklich mit dem menschlichen Körper aus - nicht nur im muskuloskelettalen Bereich, nein, in seiner Ganzheit: parietal, viszeral, kraniosakral und wie alles miteinander verknüpft ist. Die Patienten bestärken uns in der Meinung, besser zu sein, wenn auch der fundierte Beweis dazu aussteht.

Sicher, es gibt die operative Orthopädie sowie die Grenzfälle. Hand aufs Herz: Wie unterscheidet sich eine primäre Wirbelgelenksblockierung von einer Spondylodiszitis, einer bakteriellen Entzündung der Bandscheibe? Temperaturerhöhung und allgemeines Krankheitsgefühl. Eine sichere Nachweismethode im Frühstadium ist die Computer-, oder noch besser, die Kernspintomographie. Die verzögerte Einleitung einer spezifischen Therapie kann den Patienten zum Krüppel machen.

Es gibt also Fälle, in denen ein anderes Wissen erforderlich ist. Die alten Füchse unter den Osteopathen haben es längst erkannt: Wichtig ist eine wohlabgewogene Zusammenarbeit mit ausgesuchten Ärzten. Mit wem kann ich was besprechen? Denn: In Zweifelsfällen erst einmal medizinisch abklären lassen.

Sollte also die Arbeitsteilung lauten, alle funktionellen Beschwerden gehören in die Hand des Osteopathen und alles Strukturelle sowie die Grenzfälle zum Orthopäden? Schöne osteopathische Wunschvorstellung; sie geht aber völlig an der Realität unseres Gesundheitssystems vorbei. Osteopathie ist, zumindest oberflächlich betrachtet (und was sonst tun die Gesundheitspolitiker?), Luxusmedizin. Was für Möglichkeiten gibt es also in einer orthopädischen Praxis mit gut gefülltem Wartezimmer? Behandlungszeiten von 30 Minuten oder mehr scheiden aus. Nicht aber der ganzheitliche Ansatz: Schnell die Situation erfassen, auf den Punkt kommen und ein oder zwei gezielte Techniken einsetzen, auch osteopathische.

Dafür ist aber vielen Ärzten eine Ausbildung mit 1500 Stunden zu umfangreich, obwohl sie eine größere Sicherheit und Palpationsfähigkeit vermittelt. Hier setzen Ausbildungen an, die osteopathische Behandlungsmethoden nach dem Vorbild der amerikanischen Postgraduierten Kurse lehren, mit den dafür nötigen anatomischen und physiologischen Basisinformationen. Natürlich wird auch die osteopathische Denkweise gelehrt. So ist auch ein Ausbildungsgang von ca. 420 Stunden, aufbauend auf einer manualtherapeutischen Ausbildung, eine gute Basis für eine solide osteopathische Arbeit.

Um die Frage des VOD aufzugreifen: „Quo vadis, Osteopathie?“ - In Deutschland wird es auf absehbare Zeit zweigleisig weitergehen. Einerseits die langfristige Ausbildung zum Osteopathen, andererseits Ärzte und Physiotherapeuten, die in osteopathischen Verfahren weitergebildet sind. Das Gebot der Stunde ist gegenseitiges Verständnis, nicht Konfrontation.

Die Herausgeber 